\title{
Emotional numbing and pain intensity predict the development of pain disability up to one year after lateral thoracotomy
}

\author{
Joel Katz ${ }^{\mathrm{a}, \mathrm{b}, \mathrm{c}, \boldsymbol{*}}$, Gordon J.G. Asmundson ${ }^{\mathrm{d}}$, Karen McRae ${ }^{\mathrm{a}, \mathrm{c}}$, Eileen Halket ${ }^{\mathrm{a}}$ \\ a Department of Anesthesia and Pain Management, Toronto General Hospital and Mount Sinai Hospital, Toronto, ON, Canada \\ ${ }^{b}$ Department of Psychology and School of Kinesiology and Health Science, BSB 232, York University, 4700 Keele Street, Toronto, ON M3J 1 P3, Canada \\ ${ }^{c}$ Department of Anesthesia, University of Toronto, Toronto, ON, Canada \\ 'Department of Psychology and The Anxiety and Illness Behaviour Laboratory, University of Regina, Regina, SK, Canada
}

\begin{abstract}
A B S T R A C T
Little is known about the factors that predict the transition of acute, time limited pain to chronic pathological pain following postero-lateral thoracotomy. The aim of the present prospective, longitudinal study was to determine the extent to which (1) pre-operative pain intensity, pain disability, and posttraumatic stress symptoms (PTSS) predict post-thoracotomy pain disability 6 and 12 months later; and (2) if these variables, assessed at 6 months, predict 12 month pain disability. Fifty-four patients scheduled to undergo postero-lateral thoracotomy for intrathoracic malignancies were recruited before surgery and followed prospectively for one year. The incidence of chronic post-thoracotomy pain was $68.1 \%$ and $61.1 \%$ at the 6 and 12 month follow-ups, respectively. Multiple regression analyses showed that neither preoperative factors nor acute movement-evoked post-operative pain predicted 6 or 12 month pain disability. However, concurrent pain intensity and emotional numbing, but not avoidance symptoms, made unique, significant contributions to the explanation of pain disability at each follow-up (total $R^{2}=76.3 .0 \%$ and $63.9 \%$ at 6 and 12 months, respectively, both $p<0.0009$ ). The relative contribution of pain intensity decreased, while that of emotional numbing increased with time, indicating a progressive de-coupling of pain intensity and disability and a concomitant strengthening of the link between emotional numbing and disability. This suggests that pain may serve as a traumatic stressor which causes increased emotional numbing. The results also support recent suggestions that avoidance and emotional numbing constitute separate PTSS clusters. Further research is required to determine the source $(s)$ of emotional numbing after postero-lateral thoracotomy and effective interventions.
\end{abstract}

\section{Introduction}

Most patients who undergo surgery do not develop chronic post-surgical pain (CPSP). They recover uneventfully and within weeks resume everyday activities. The typical course of recovery, however, is not enjoyed by many patients whose pain does not abate. The scale of the problem is documented by the growing awareness of CPSP in the surgical community (Kehlet et al., 2006; Macrae, 2001a, 2001b; Perkins and Kehlet, 2000). A prospective study of approximately 5000 patients estimated the incidence of acute neuropathic pain following surgery to be $1-3 \%$. A one year follow-up showed that $56 \%$ of patients with neuropathic pain reported ongoing pain (Hayes et al., 2002). Considering the total

\footnotetext{
* Corresponding author. Address: Department of Psychology and School of Kinesiology and Health Science, BSB 232, York University, 4700 Keele Street, Toronto, ON M3J 1P3, Canada. Tel.: +416 736 2100x40557; fax: +416 7365814. E-mail address: jkatz@yorku.ca (J. Katz).
}

number of patients worldwide who undergo surgery every year, it is not surprising that almost $25 \%$ of patients seen at chronic pain treatment centers are referred for CPSP (Crombie et al., 1998).

One of the most intractable CPSPs arises after postero-lateral thoracotomy for lung cancer. Given the poor prognosis and low survival rates associated with lung cancer (Mountain, 1997; Mountain and Dresler, 1997), the main priority is to optimize quality of life (Cella, 2004; Pearman, 2008). However, quality of life among survivors is compromised by an unacceptably high occurrence of chronic post-thoracotomy pain. The one-year incidence of chronic post-thoracotomy pain is between $21 \%$ (Ochroch et al., 2002) and $61 \%$ (Perttunen et al., 1999), with most estimates around 50\% (Gotoda et al., 2001; Ju et al., 2008; Katz et al., 1996).

Very little is known about the mechanisms underlying development and maintenance of chronic post-thoracotomy pain. Biopsychosocial models acknowledge the important interplay between biomedical and psychosocial variables in chronic neuropathic pain and disability in other surgical patient populations (Hanley et al., 
2004, 2007; Jensen et al., 2002). However, there have been no studies of risk factors associated with development of chronic post-thoracotomy pain and disability.

Recent data (Asmundson et al., 2002) show high co-morbidity between chronic pain and post-traumatic stress disorder (PTSD). PTSD usually occurs after exposure to a situation or event perceived as threatening to one's physical or emotional integrity. According to the Diagnostic and Statistical-Manual of Mental Disorders-IV Text Revision (DSM-IV TR, American Psychiatric Association, 2000), the diagnostic criteria for PTSD include three symptom clusters - (1) re-experiencing (e.g., nightmares), (2) avoidance (e.g., of activities associated with the event) and emotional numbing (e.g., feeling detached from others), and (3) hyper-arousal (e.g., jumpiness). The lifetime prevalence for PTSD ranges from $1 \%$ to $14 \%$ but may be as high as $58 \%$ for at-risk individuals (e.g., soldiers, victims of assault) (American Psychiatric Association, 2000). Many more individuals not meeting full diagnostic criteria may suffer with partial or subthreshold PTSD (Mylle and Maes, 2004). Reasons for the high co-occurrence of chronic pain and post-traumatic stress symptoms (PTSS) include mutual maintenance of overlapping symptoms and shared vulnerability mechanisms (Asmundson et al., 2002).

The aim of the present prospective, longitudinal study was to determine the extent to which (1) pre-operative pain intensity, pain disability, and PTSS predict post-thoracotomy pain disability 6 and 12 months later; and (2) if these variables, assessed at 6 months, predict 12 month pain disability. Based on suggestions linking emotional numbing with endogenous opioid dysregulation (Foa et al., 1992; Glover, 1993), evidence that avoidance and emotional numbing represent distinct PTSD symptoms clusters (Asmundson et al., 2004), and evidence that pain severity and emotional numbing symptoms predict quality of life in chronic pain patients (Clapp et al., 2008), we hypothesized that both pain intensity and emotional numbing, but not avoidance, would predict long term post-thoracotomy pain disability.

\section{Methods}

\subsection{Participants}

Fifty-four patients scheduled to undergo postero-lateral thoracotomy for intrathoracic malignancies were recruited approximately one week before surgery and followed prospectively for one year.

\subsection{Procedure}

The study was approved by the Research Ethics Board at the Toronto General Hospital, University Health Network and by the Human Participants Review Committee at York University. Data for the present study were collected as part of a larger prospective research project examining various facets of fear and anxiety in patients undergoing major surgery. Patients who underwent posterolateral thoracotomy for intrathoracic malignancies constituted the sample for the present paper.

Eligible patients met the following inclusion criteria: undergoing major surgery; scheduled to be admitted for at least 2 days after surgery; to receive intravenous or epidural patient controlled analgesia post-operatively for at least 2 days; $18-60$ years of age; able to read and understand English, not enrolled in another research study.

Patients were approached in the hospital pre-admission unit approximately one week before surgery by a research assistant or research nurse not involved with the patients' clinical care. The nature and purpose of the study was explained to them.
Patients who agreed to participate in the study signed an informed consent form after which they completed a demographic information form and a battery of questionnaires including an assessment of pre-operative pain intensity. At that time, we also obtained pressure pain thresholds $\left(\mathrm{kg} / \mathrm{cm}^{2}\right)$ by applying a Fischer (1987) algometer (Pressure Threshold Meter, Pain Diagnostics and Thermography, Great Neck, NY, USA) to the side of the chest ipsilateral to the site of proposed surgery and at control sites on the left and right upper legs (anterior aspect midway between the knee and hip).

Each patient received a general anesthetic in accordance with hospital clinical practice. In addition, patients received iv PCA morphine (0.5-2.0 mg bolus doses, lock out $5 \mathrm{~min}, 40 \mathrm{mg}$ maximum in $4 \mathrm{~h}$ ), iv PCA hydromorphone (0.1-0.4 mg bolus doses, lock out $5 \mathrm{~min}, 10 \mathrm{mg}$ maximum in $4 \mathrm{~h}$ ), epidural infusion with PCEA ( $0.1 \%$ bupivacaine and $0.015 \mathrm{mg} / \mathrm{ml}$ hydromorphone), or both an epidural infusion of ropivacaine $0.2 \%$ and iv opioid via PCA. Patients also received adjuvant analgesics with acetaminophen and/ or ketorolac. Analgesic consumption was downloaded from the PCA and PCEA pumps and the cumulative dose at $48 \mathrm{~h}$ was calculated from hard copy records. Pain at rest and after standardized movement was measured at $24 \mathrm{~h}$ and $48 \mathrm{~h}$ after surgery.

Patients were followed up by telephone at 6 and 12 months after surgery and were re-administered the same questionnaires they had completed at the pre-admission visit. Patients were telephoned a maximum of three times. A voice message was left on the third call. Patients were considered lost to follow-up if they could not be reached and did not return the call.

Hospital and thoracic surgery department records were reviewed to determine the presence of a local recurrence of the malignancy at the time of the 6 and 12 month follow-ups. Patients are routinely seen by their surgeon 6 and 12 months after surgery, and additionally are seen more often should they develop new symptoms. Routine assessment included physical examination and radiologic assessment with X-ray and/or CT scan of the chest. Local recurrence was defined as a new mass in the chest consistent with tumor, bony invasion of the thoracic spine or a pleural effusion containing malignant cells.

Data from the following questionnaires measured pre-operatively and at the 6 and 12 month follow-up interviews were analyzed for the present paper.

\subsection{Measures}

\subsubsection{Pain intensity}

Pain intensity was measured with a numeric rating scale (NRS). The NRS consists of a series of numbers ranging from 0 to 10 with endpoints representing the most extreme pain experiences $(0=$ no pain and $10=$ worst possible pain). Patients were asked to choose the number that best corresponded to the intensity of their pain. We assessed average daily pain (NRS-A) at the pre-assessment visit ("On the days that you feel pain, what is the average intensity of your pain"). We assessed rest pain and movement-evoked pain (NRS-M) on days 1 and 2 after surgery ( $24 \mathrm{~h}$ and $48 \mathrm{~h}$ after surgery). The latter measure involved having the patient sit up in bed from a lying position and then provide a pain rating. We also assessed average and worst pain at the 6 month and 12 month follow-ups. The NRS has demonstrated good reliability and validity and has been shown to be sensitive to change following pharmacological intervention (Katz and Melzack, 1999).

\subsubsection{Pain disability index (PDI)}

The PDI (Pollard, 1984) is a self-report questionnaire that measures the extent to which pain interferes with seven daily, psychosocial activities and life domains, including (1) family/home responsibilities, (2) recreation, (3) social activity, (4) occupation, 
(5) sexual behavior, (6) self-care, and (7) life-support activity. Each domain is rated on an 11-point scale ranging from $0=$ no disability to $10=$ total disability. The total score ranges from 0 to 70 . The PDI has high internal consistency $(.86)$, modest test-retest reliability (.44), good concurrent validity based on significant correlations with objective measures of pain related disability (Chibnall and Tait, 1994; Tait et al., 1990, 1987). The PDI is sensitive to change in response to pre-operative analgesic treatment for up-to 3 weeks after surgery (Katz and Cohen, 2004). The PDI showed excellent internal consistency at all three time points (Cronbach's alpha ranged between 0.92 and 0.97 ).

2.3.3. Post-traumatic stress disorder checklist-civilian version ( $P C L-C)$ The PCL-C (Weathers et al., 1993) is a 17-item self-report measure. Each item is a statement based on the current DSM-IV TR (American Psychiatric Association, 2000) symptoms for PSTD. Respondents are asked to indicate how much they have been bothered by each symptom over the past month, on a scale from $1=$ not at all to $5=$ extremely. The questionnaire produces a total score as well as subscale scores for re-experiencing, avoidance and emotional numbing, and hyper-arousal. The current DSM-IV TR convention of combining avoidance and numbing into a single symptom cluster may not be the most accurate conceptualization of PTSS (Asmundson et al., 2004). Recent conceptual (Asmundson et al., 2004; Foa et al., 1992) and empirical work from factor analytic (Asmundson et al., 2000), treatment outcome (Taylor et al., 2001, 2003), and correlational (Clapp et al., 2008; Felmingham et al., 2002) studies suggests that avoidance and emotional numbing constitute separate symptom clusters. For example, Clapp et al. (2008) found that pain severity and emotional numbing, but not the other PTSD symptom clusters, were significantly negatively related to role functioning in patients with ongoing pain subsequent to motor vehicle injury. Based on this emerging and empirically supported conceptualization of PTSS, we used a scoring procedure that separates avoidance and emotional numbing items. The emotional numbing subscale was calculated by summing the responses to items 8 through 12 and avoidance subscale was calculated by summing the responses to items 6 and 7. Examples of emotional numbing items include: Item 11, "Feeling emotionally numb or being unable to have loving feelings for those close to you?" and Item 12, "Feeling as if your future somehow will be cut short?". The two items that make up the avoidance subscale are Item 6 , "Avoiding thinking about or talking about a stressful experience from the past or avoiding having feelings related to it?", and Item 7. "Avoiding activities or situations because they reminded you of a stressful experience from the past?" Cut-off scores that reliably predict PTSD diagnosis range between 44 (Weathers et al., 1993) and 50 (Blanchard et al., 1996). Test-retest reliability for the PCL-C over a 2-3 day retest interval is 0.96 (Weathers et al., 1993). The PCL-C yields an adequately high overall diagnostic efficiency and is both reliable and valid (Weathers et al., 1993). The PCL-C showed excellent internal consistency at all three time points (Cronbach's alpha ranged between 0.90 and 0.94).

\subsection{Data analysis}

Data were analyzed using Statistical Package for the Social Sciences (version 15.0.1. for Windows, SPSS Inc., Chicago, IL, USA). Pearson correlation coefficients were calculated to examine bivariate relationships among variables. Multivariate association was examined using regression analyses to predict 6 and 12 month pain disability scores. To determine the eligible demographic, medical, and pain covariates to enter into the regression equations, we computed the association between the outcome variables ( 6 and 12 month PDI scores) and age, sex, education level, duration of pre-operative pain, pre-operative mechanical pressure pain thresholds, surgical procedure, American Society of Anesthesiologists physical status classification, duration of surgery, perioperative epidural use, cumulative dose of post-operative analgesia, chemotherapy, radiation therapy, and cancer recurrence. None of the above variables was significantly associated with either outcome variable (all $p>0.05$ ).

Therefore, for the 6 and 12 month follow-ups, we conducted the following three-step multiple regression analyses predicting follow-up PDI scores: In step 1, we entered pre-operative PDI scores, PTSS (PCL-C emotional numbing or avoidance scores), NRS-A pain intensity scores, and mean NRS movement-evoked pain intensity scores on days 1 and 2 after surgery. In step 2, we entered NRS-A pain intensity scores at the follow-up. In the final step, we entered follow-up PCL-C symptoms (emotional numbing or avoidance scores). Thus, we were interested in determining the proportion of variance in follow-up pain disability explained by concurrent (i.e., 6 or 12 month) PTSS (PCL-C emotional numbing or avoidance scores) after controlling for pre-operative pain intensity and disability, pre-operative PTSS (PCL-C emotional numbing or avoidance scores) and acute post-operative movement-evoked pain.

We calculated a third three-step multiple regression analysis predicting 12 month follow-up PDI scores from 6 month data. In step 1, we entered 6 month NRS-A pain intensity, PDI, and PCLemotional numbing (or avoidance) scores. In step 2, we entered 12 month follow-up NRS-A pain intensity scores and in the final step, we entered 12 month follow-up PCL-C emotional numbing (or avoidance) scores. Thus, for this analysis, we were interested in determining the proportion of variance in pain disability at 12 months explained by 12 month PTSS symptoms (emotional numbing or avoidance) after controlling for 6 month NRS-A pain intensity, pain disability, PTSS (emotional numbing or avoidance), and 12 month pain intensity.

Finally, we ran separate one-way linear mixed models repeated measures ANOVAs comparing mean PDI, PCL-C emotional numbing, $\mathrm{PCL}-\mathrm{C}$ avoidance, and NRS-A pain intensity scores obtained at the initial assessment and the 6 and 12 month follow-ups. A significant main effect was follow-up with Bonferroni adjusted post hoc comparisons to evaluate the pattern of change over time.

\section{Results}

\subsection{Recruitment and retention of patients}

Fig. 1 shows the flow chart outlining recruitment and retention of study patients. Patients were recruited between July 2003 and July 2006 during which time 351 patients were scheduled to undergo lateral thoracotomy. Of these, 215 did not meet the inclusion criteria (208 were older than 60 or younger than 18 years of age, five were not proficient in English, one was not to receive PCA, and one was already participating in another study). Of the remaining 136 patients, 31 refused to participate for various reasons and 51 were missed by study personnel ( 29 had their surgery or appointment rescheduled without notice, for 10 patients study personnel were not available, for nine patients study personnel were not informed of the patient's availability, two patients were incorrectly deemed ineligible, and one patient did not show up at the pre-admission unit).

Fifty-four patients were recruited into the study. One patient withdrew consent prior to surgery. Surgery was cancelled for another patient. Of the remaining 52 patients who underwent thoracotomy, 47 (90.4\%; 24 male, 23 female) were contacted and followed up 6 months after surgery, three had died and two were lost to follow-up. Twelve months after surgery, 36 (69.2\%; 14 male, 22 female) patients were followed up. Between the 6 and 12 month follow-ups, seven patients had died (total 10 deaths), five were lost 


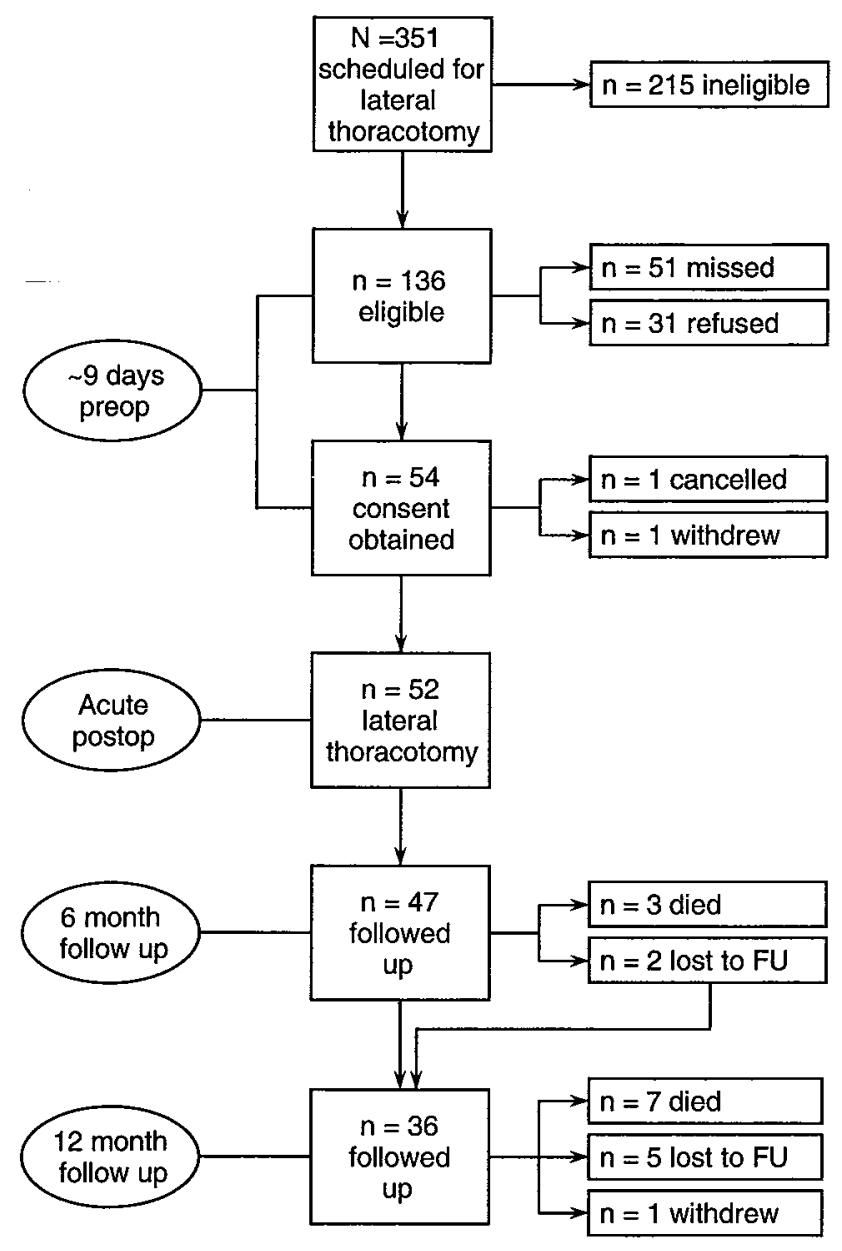

Fig. 1. Chart of patient flow through the study. See text for details.

to follow-up (total five lost to follow-up as the two patients who were lost to follow-up at 6 months were reached for the 12 month follow-up) and one refused to be interviewed.

\subsection{Demographic and clinical variables}

Table 1 shows the demographic and clinical variables describing the sample of 52 patients at the initial assessment approximately one week before surgery. Table 1 also contains clinical variables including the surgical procedure and the doses of drugs received by the epidural and iv routes over the course of the first two post-operative days. Twenty-one patients (38.8\%) reported preoperative pain. Twelve patients received iv PCA (morphine or hydromorphone), 35 received epidural PCA (bupivacaine and hydromorphine), three received iv PCA and a background infusion of epidural ropivacaine, one patient was started on epidural PCA and was switched to iv PCA and one patient received iv hydromorphone. Table 2 shows scores on the psychosocial measures at the initial assessment and follow-ups.

\subsection{Changes in PTSS, pain intensity, and pain disability over time}

Table 2 shows mean \pm sd NRS-A pain intensity scores and the psychosocial variables at the pre-admission visit and the 6 and 12 month follow-ups. A series of linear mixed model repeated measures ANOVAs revealed a significant main effect of time for the PCL-C total score $(F(2,41.029)=3.576, p=0.037)$ and PCL-C avoidance symptoms $(F(2,60.096)=5.170, p=0.008)$, but not for
PCL-C emotional numbing, pain disability, or NRS-A pain intensity (all $p>0.216$ ), indicating that for the latter variables, there was no significant change from baseline to the 12 month follow-up. Bonferroni adjusted post hoc comparisons showed that both the PCL$C$ total score $(p=0.030)$ and avoidance score $(p=0.012)$ decreased significantly from the baseline pre-operative assessment to the 12 month follow-up.

\subsection{Pre-operative prediction of 6 month pain disability}

At the 6 month follow-up, 32 of the 47 (68.1\%) patients reported CPSP (mean \pm sd NRS average pain intensity: $3.94 \pm 2.0$; NRS worst pain intensity: $6.31 \pm 2.9$ ) located predominantly at the thoracotomy scar and chest wall. Of those with pain, 15 patients (46.9\%) described it as constant. The remainder described the pain as periodic or brief with most (74\%) indicating that it occurred on at least a daily basis. Twenty-one (65.6\%) reported taking medication for the pain. Eleven (34.4\%) patients had actively sought treatments for the pain including referral to a pain clinic, relaxation training, acupuncture, physiotherapy, and massage therapy. Eleven $(34.4 \%)$ reported having resumed normal or modified work activities. Nine of the $47(19.1 \%)$ patients had a documented local recurrence of the malignancy at the time of the 6 month follow-up.

Table 3 shows the correlation matrix of the regression model variables predicting 6 month pain disability scores. Regression analysis showed that the pre-operative factors and acute movement-evoked pain intensity on days 1 and 2 after surgery (step $1: R^{2}=0.116, p=0.257$ ) did not significantly predict 6 month pain disability (Table 4). In contrast, the addition of 6 month pain intensity scores in step $2\left(\Delta R^{2}=0.604, p=0.0009\right)$ was a significant predictor of 6 month pain disability. Importantly, after controlling for all variables in the model, 6 month emotional numbing scores accounted for a small, but significant proportion of variance in 6 month pain disability scores (step 3: $\Delta R^{2}=0.042, p=0.011$ ). In the final model, only 6 month pain intensity scores (beta $=0.727$; $p=0.0009$ ) and 6 month emotional numbing (beta $=0.245$; $p=0.011$ ) contributed significantly to the prediction of 6 month pain disability scores $\left(F(6,40)=21.48, p=0.0009\right.$; total $R^{2}=0.763$ ).

In contrast to the emotional numbing symptoms, 6 month avoidance symptoms (beta $=-0.007 ; p=0.942 ; \Delta R^{2}=0.000, p=0$. 942) failed to contribute significantly to the explanation of 6 month pain disability scores.

\subsection{Pre-operative prediction of 12 month pain disability}

At the 12 month follow-up, 22 of the $36(61.1 \%)$ patients reported CPSP (mean \pm sd NRS average pain intensity: $3.90 \pm 2.0$; NRS worst pain intensity: $6.89 \pm 2.9$ ) located predominantly at the thoracotomy scar and chest wall. Of those with pain, 7 patients (31.8\%) described it as constant. The remainder described the pain as periodic or brief with most (60\%) indicating that it occurred on at least a daily basis. Eleven (50\%) reported taking medication for the pain. Seven (31.8\%) patients had actively sought treatments for the pain including relaxation, acupuncture, physiotherapy, and massage therapy. Thirteen (59.1\%) reported having resumed normal or modified work activities. Five of the $36(13.8 \%)$ patients had a documented local recurrence of the malignancy at the time of the 12 month follow-up.

Table 5 shows the correlation matrix of the regression model variables predicting 12 month pain disability scores. Similar to the 6 month data, regression analysis showed that pre-operative factors and acute movement-evoked pain intensity on days 1 and 2 after surgery (step $1: R^{2}=0.148, p=0.275$ ) did not significantly predict 12 month pain disability (Table 6 ). The addition of 12 month pain intensity scores in step $2\left(\Delta R^{2}=0.291, p=0.0009\right)$ was a significant predictor of 12 month pain disability, although, 
Table 1

Demographic and clinical variables.

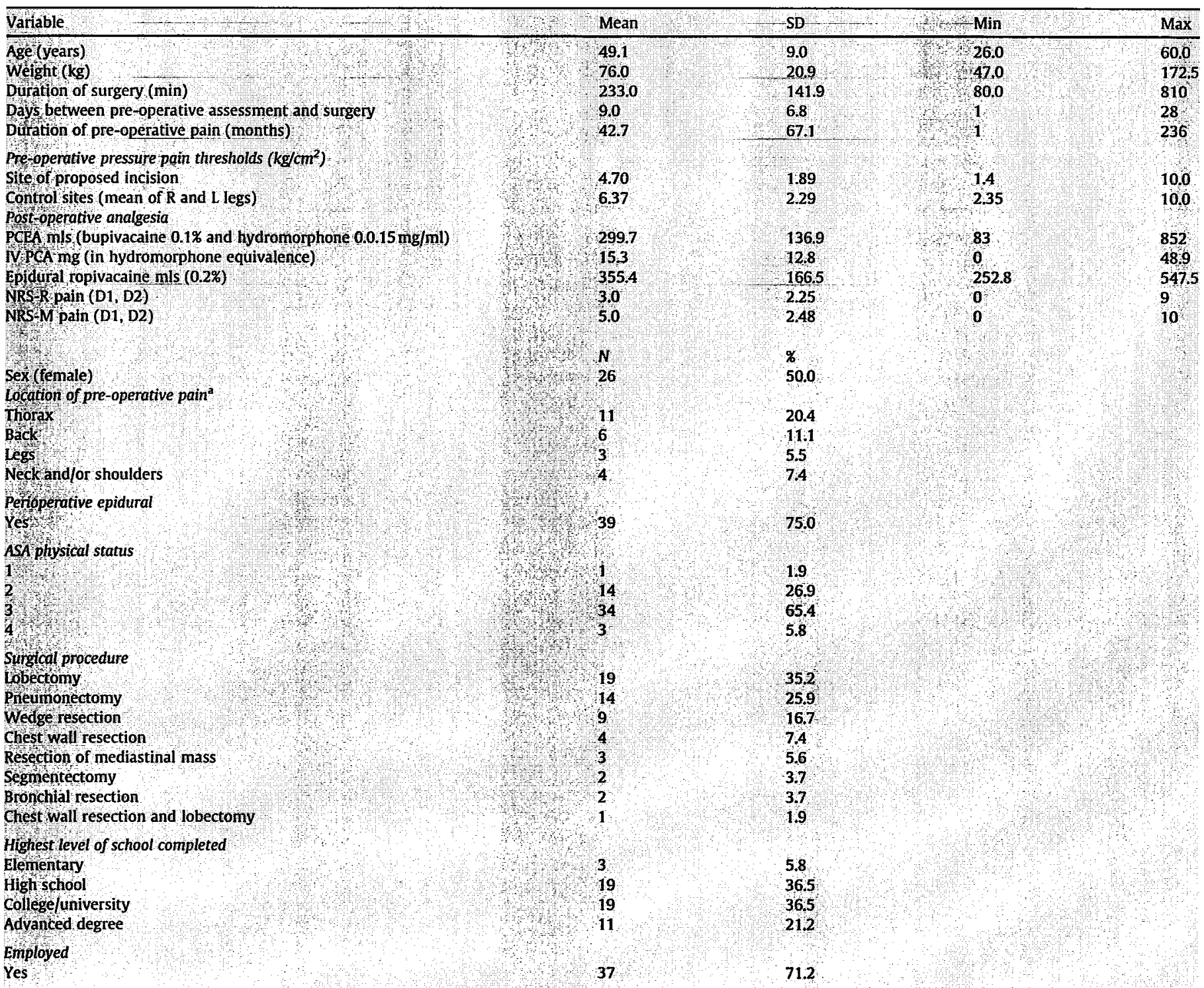

ASA: American Society of Anesthesiologists, NRS-M pain (D1, D2) refers to the mean movement-evoked pain intensity averaged across days 1 and 2 postop as measured by a numeric rating scale. Mean NRS-R pain (D1, D2) refers to the mean intensity of pain at rest averaged across days 1 and 2 postop as measured by a numeric rating scale.

${ }^{a}$ The total exceeds the number of patients $(N=21)$ who had pre-operative pain since some patients reported pain at multiple locations.

notably, less so (by $\sim 31 \%$ ) than at 6 months. Finally, after controlling for all variables in the model, 12 month emotional numbing

Table 2

Pain and psychosocial variables at the pre-operative and follow-up assessments.

\begin{tabular}{|c|c|c|c|c|c|c|}
\hline \multirow{3}{*}{ Variable } & \multicolumn{2}{|c|}{ Pre-operative } & \multirow{2}{*}{\multicolumn{2}{|c|}{$\frac{6 \text { month FU }}{N=47}$}} & \multicolumn{2}{|c|}{12 month Fu } \\
\hline & \multicolumn{2}{|l|}{$N=52$} & & & \multicolumn{2}{|c|}{$N=36$} \\
\hline & Mean & SD & Mean & SD & Mean & SD \\
\hline NRS $A$ pan intensi & 23 & 34 & 2.7 & 2.5 & 2.4 & 25 \\
\hline PDI total & 19.7 & 172 & 23.4 & 225 & 153 & 202 \\
\hline PCL-C total & 33.8 & 12.5 & 30.6 & 13.8 & 26.8 & 106 \\
\hline PCL-C numbing & 9.6 & 4.1 & 9,2 & 4,9 & 79 & 3.9 \\
\hline 'PCL-C avoidance & 3.6 & 2.0 & 32 & 18 & 27 & 1.1 \\
\hline
\end{tabular}

NRS-A: numeric rating scale-average pain intensity; $\mathrm{PDI}$ : pain disability index; $\mathrm{PCL}$ $C:$ post-traumatic stress disorder checklist-civilian version.

$p=0.030$.

b $p=0.012$ for pre-operative versus 12 month FU. scores accounted for a significant proportion of variance in 12 month pain disability scores (step 3: $\Delta R^{2}=0.200, p=0.0009$ ) and considerably more variance $(\sim 16 \%)$ than at 6 months. However, in the final model, only 12 month emotional numbing (beta $=0.558 ; p=0.0009$ ) contributed significantly to the prediction of 12 month pain disability scores $(F(6,29)=8.56, p=$ 0.0009 ; total $R^{2}=0.639$ ).

In contrast to the emotional numbing symptoms, 12 month avoidance symptoms (beta $=-0.012 p=0.934 ; \Delta R^{2}=0.000$, $p=0.934$ ) failed to contribute significantly to the explanation of 12 month pain disability scores.

\subsection{Six month prediction of 12 month pain disability}

Table 7 shows the correlation matrix of the regression model variables predicting 12 month pain disability scores from 6 and 12 month follow-up data. All variables were significantly related 
Table 3

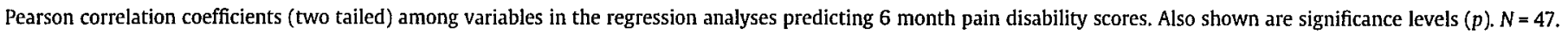

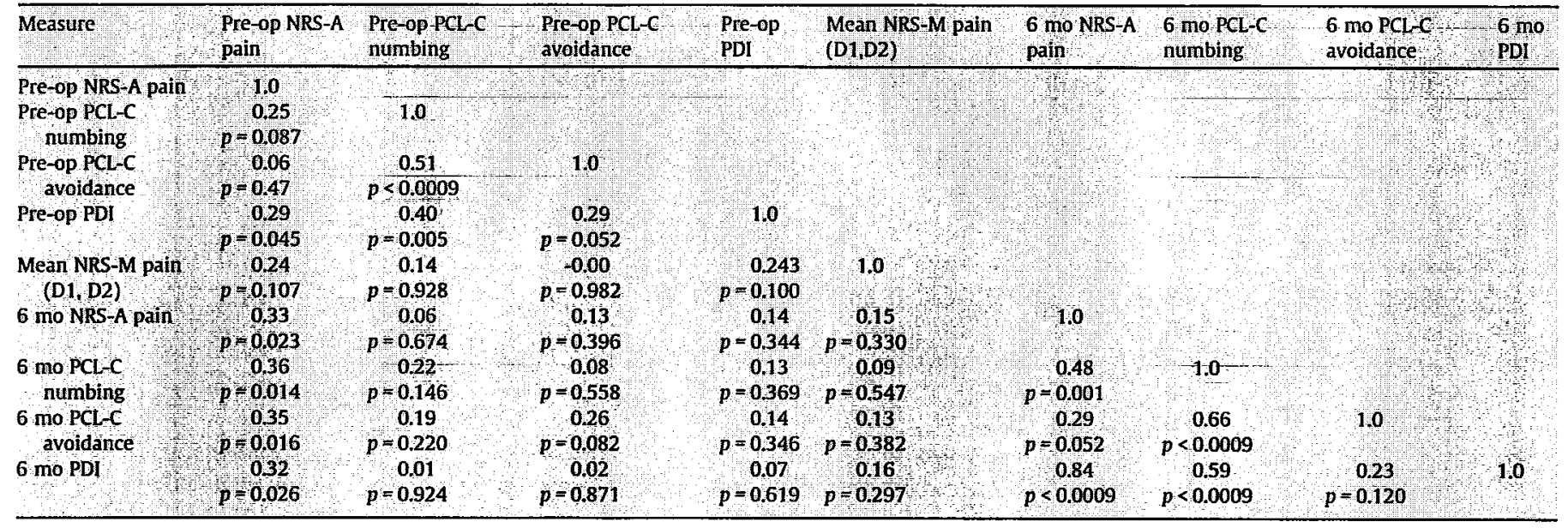

Abbreviations as in Table 2.

Table 4

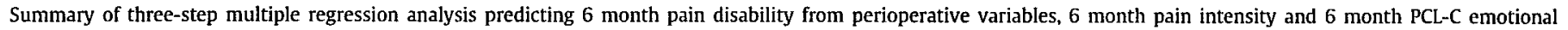
numbing scores $(N=47)$. Abbreviations as in Tables 2 and 3.

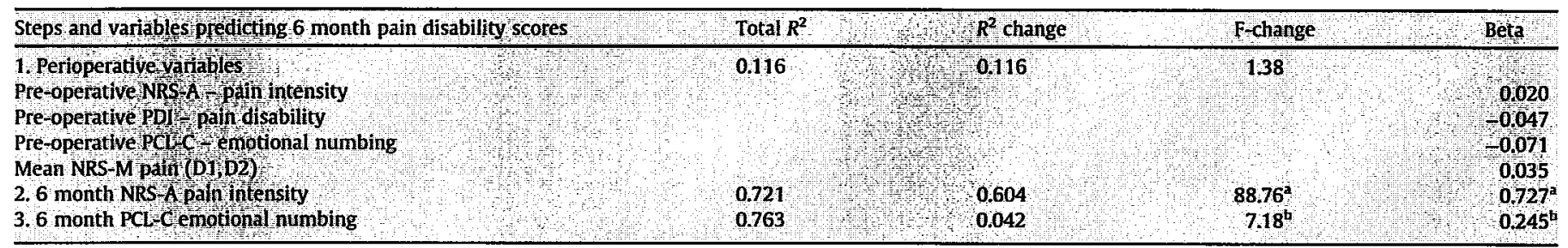

a $p<0.0009$.

b $p=0.011$.

Table 5

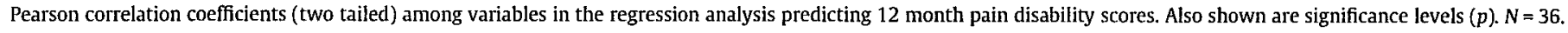

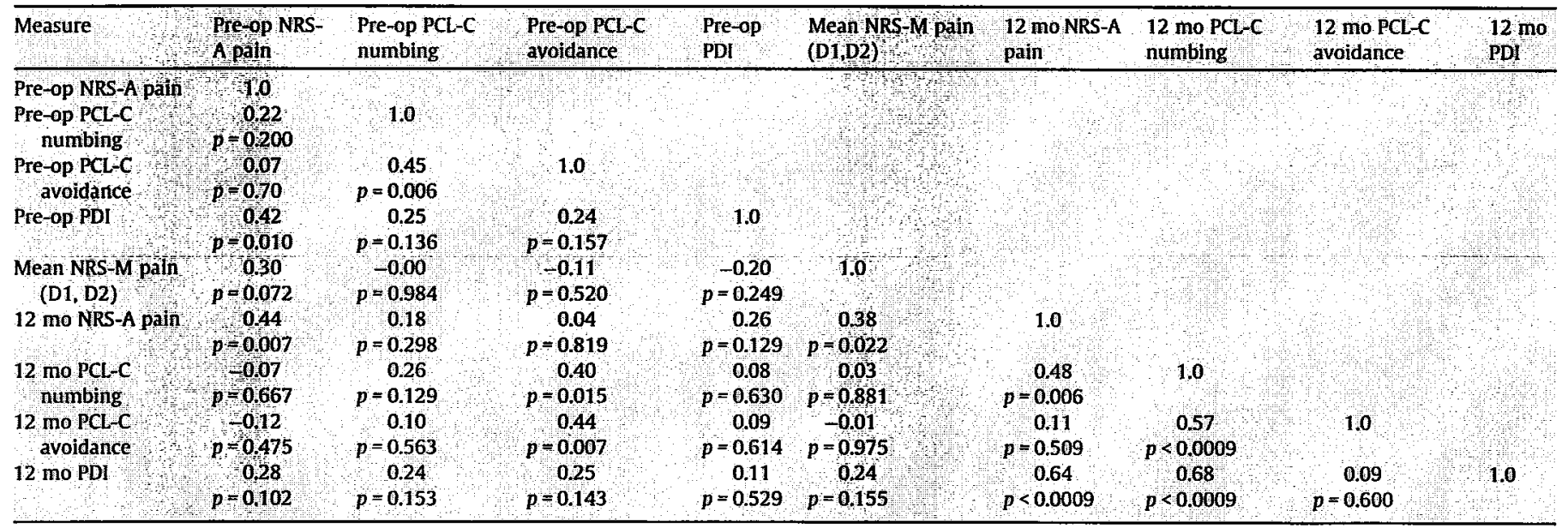

Abbreviations as in Tables 1 and 2.

to the main outcome variable (12 month PDI scores) as well as to one another. Results of the regression analysis (Table 8 ) showed that after controlling for 6 month variables (pain intensity, emotional numbing, and pain disability) (step 1: $R^{2}=0.559$, $p=0.0009$ ), 12 month pain intensity scores (step $2: \Delta R^{2}=0.082$, $p=0.016$ ) and 12 month emotional numbing scores (step 3: $\Delta R^{2}=0.095, p=0.004$ ) made significant, independent contributions to the prediction of 12 month pain disability scores. The final model shows that 6 month pain disability scores (beta $=0.652$; $p=0.006$ ), 12 month pain intensity (beta $=0.265 ; p=0.047$ ), and 12 month emotional numbing (beta $=0.372 ; p=0.004$ ) contributed significantly to the prediction of 12 month pain disability scores $\left(F(5,28)=15.51, p=0.0009\right.$; total $\left.R^{2}=0.735\right)$.

In contrast to the above regression analysis, neither 6 month (beta $=-0.176 ; p=0.189 ; \Delta R^{2}=0.002, p=0.710$ ) nor 12 month avoidance symptoms (beta $=0.136 ; p=0.248 ; \Delta R^{2}=0.017, p=0$. 
Table 6

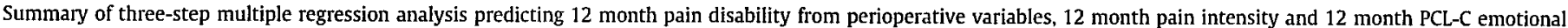
numbing scores $(N=36)$. Abbreviations as in Tables 1 and 2

\begin{tabular}{|c|c|c|c|c|}
\hline Steps and Variables Predicting 12 Month Pain Disability Scores & Total $\mathbf{R}^{2}$ & $\mathbf{R}^{2}$ change & FChange & Beta \\
\hline 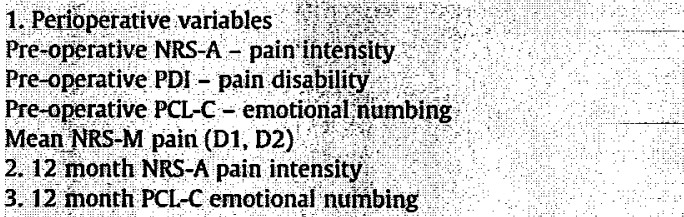 & $\begin{array}{l}0.148 \\
0.439 \\
0.639\end{array}$ & 0,148 & $\begin{array}{l}15.55^{\mathrm{a}} \\
16.09^{\mathrm{a}}\end{array}$ & $\begin{array}{r}0209 \\
0127 \\
0033 \\
0,076 \\
0,299 \\
0,558\end{array}$ \\
\hline
\end{tabular}

${ }^{a} p<0.0009$

Table 7

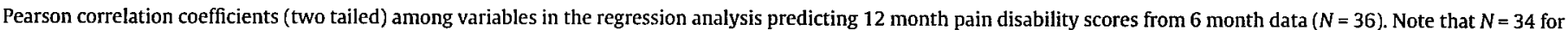

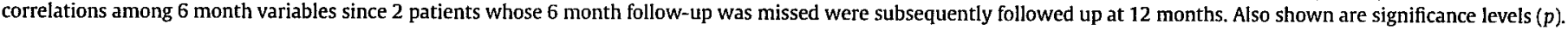

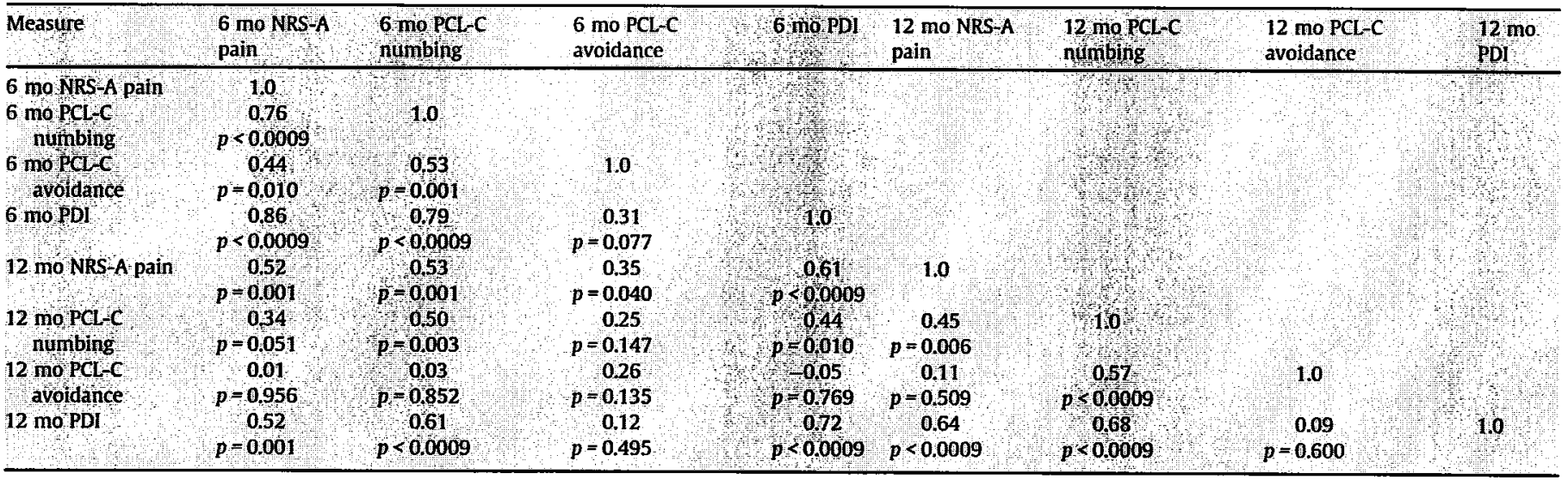

Abbreviations as in Table 2.

Table 8

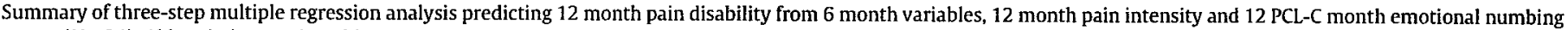
scores $(N=34)$. Abbreviations as in Table 2.

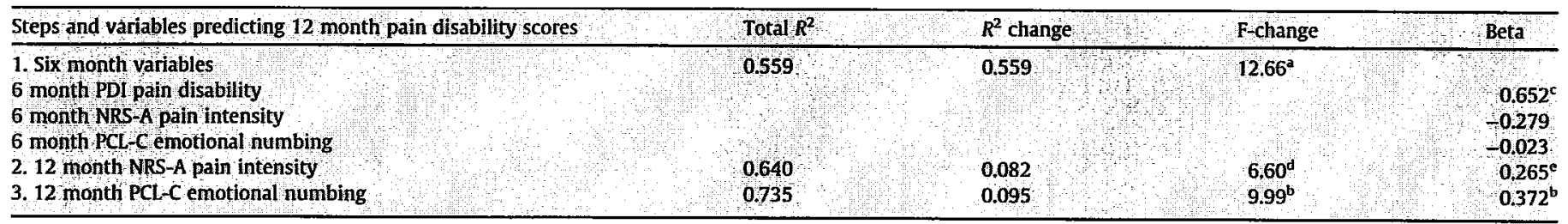

$$
\begin{aligned}
& \text { d } p<0.0009 . \\
& \text { b } p=0.004 . \\
& \text { c } p=0.006 . \\
& \text { d } p=0.016 . \\
& \text { e } p=0.047 .
\end{aligned}
$$

248) contributed significantly to the explanation of 12 month pain disability scores.

\section{Discussion}

The results of the present study are consistent with previous work showing a high rate of chronic post-thoracotomy pain (Dajczman et al., 1991; Katz et al., 1996; Maguire et al., 2006; Ochroch et al., 2002; Perttunen et al., 1999; Pluijms et al., 2006; Senturk et al., 2002). The 6 month and one year incidence rates were $68.1 \%$ and $61.1 \%$, respectively, which is in line with most other prospective studies. Consistent with previous research the intensity of the pain was, on average, moderate and occurred on at least a daily basis for the majority of patients. To our knowledge, this is the first study to measure pain disability in patients after lateral thoracotomy. Pain disability scores were, on average, low and compare well with scores reported by chronic low-back pain outpatients several months post-treatment (Tait et al., 1987) and those who were working full-time (Pollard, 1984).

The results of the present study show that $\sim 75 \%$ of the variance in 12 month pain disability scores can be explained by a combination of 6 and 12 month variables, notably, 6 month pain disability, and 12 month pain intensity and emotional numbing. While it is true that pain disability scores were, on average, low at the three assessments, the relative contribution to pain disability of pain intensity and emotional numbing appears to change with time. 
The pattern of results differed for the 6 and 12 month follow-up regression analyses; concurrent pain intensity and emotional numbing contributed significantly to the prediction of pain disability (Tables 4 and 6, respectively) at 6 months, but only 12 month emotional numbing predicted 12 month pain disability. In addition, the relative contribution of concurrent pain intensity to concurrent pain disability dropped from $60 \%$ at 6 months to $29 \%$ at 12 - months (a difference of $31 \%$ ) while that of emotional numbing increased from $4.2 \%$ at 6 months to $20 \%$ at 12 months (a difference of $15.8 \%)^{1}$

These data suggest that as time from surgery increases from 6 to 12 months, pain intensity and pain disability become progressively de-coupled while the relationship between emotional numbing and pain disability becomes stronger. One possible explanation for this pattern of findings might be that pain itself serves as a traumatic stressor which causes increased emotional numbing. For example, the post-thoracotomy pain may serve as a constant reminder of the cancer diagnosis or it may be interpreted as a recurrence of disease both of which would ultimately lead to increased emotional numbing and pain disability. Biological explanations of the link between pain and emotional numbing have implicated the endogenous opioid system (Foa et al., 1992). However, the studies that have been conducted to date show that CSF (Baker et al., 1997) and plasma (Hamner and Hitri, 1992; Hoffman et al., 1989) concentrations of beta-endorphin are significantly higher in patients with PTSD compared with controls. These studies have not examined the relationship between beta-endorphin concentrations and emotional numbing per se. Nevertheless, the results of the present study seem inconsistent with the betaendorphin hypothesis given the positive correlations between pain intensity and emotional numbing.

It is notable that pre-operative levels of pain intensity, pain disability, emotional numbing and acute movement-evoked pain did not contribute significantly to later pain disability, either at 6 months (Table 4 ) or at 12 months (Table 6). In addition, peri-operative epidural use and dose of post-operative analgesics did not correlate with 6 or 12 month pain disability scores. These results are not consistent with two studies showing that perioperative epidural use reduces the incidence and/or intensity of chronic post-thoracotomy pain (Ochroch et al., 2002; Senturk et al., 2002).

In contrast to the lack of a relationship between follow-up pain disability and the perioperative factors noted above, 6 month pain disability, and 12 month pain intensity and emotional numbing (Table 8), contributed significantly to the prediction of 12 month pain disability. Taken together, these data suggest that the main source of late pain disability (e.g., at 12 months) stems from post-surgical factors with little to no contribution from pre-operative factors or acute post-operative movement-evoked pain. This appears to be inconsistent with previous research showing that acute movement-evoked pain in the hours and days after surgery predicted the development of post-thoracotomy pain 18 months later (Katz et al., 1996). However, it is important to note that the present study found a significant univariate association $(r=0.38$, $p=0.022$ ) between acute movement-evoked pain intensity in the first two days after surgery and 12 month pain intensity scores (Table 5). In addition, the univariate relationship between pre-operative pain intensity and 12 month pain intensity was also significant $(r=0.44, p=0.007)$. However, when these variables were considered in a multivariate context, which was not done in previous research (Katz et al., 1996), they did not contribute significantly to the prediction of pain disability.

\footnotetext{
1 This pattern of results was confirmed when we ran the 6 month regression equations using only those patients reached at the 12 month follow-up indicating that the relative changes in variance from 6 to 12 months explained by pain intensity and emotional numbing are not a cohort effect.
}

Emotional numbing is regarded as a prominent feature of the more global phenomenon of dissociation (Foa et al., 1992) and peri-traumatic dissociation has been associated with an increased risk of developing PTSD (Shalev et al., 1996). It is interesting to note that in the present study, the larger contribution to 12 month pain disability was not pre-operative emotional numbing but emotional numbing at the 6 month assessment. As noted above, this suggests that the development of 12 month pain disability is more dependent on post-operative factors (i.e, occurring within 6 months of surgery) and less on pre-operative pain intensity or emotional numbing associated with pre-operative factors (e.g., anticipation of surgery or a cancer diagnosis).

The preceding discussion raises the issue of the source(s) of emotional numbing and other PTSD symptoms at the three time points. We did not assess the nature of the traumatic stressors that contributed to the PCL-C total and subscale scores. Although there has not been an evaluation of PTSD symptoms in post-thoracotomy cancer patients, studies of patients with other types of cancer suggest that the emotional numbing may arise from living with pain, which may serve as a reminder of the cancer (Bender et al., 2008), living with a diagnosis of cancer (Kangas et al., 2005) as well as the threat of and/or a recurrence of cancer (Black and White, 2005). It is also possible that the emotional numbing is, in part, due to a previous unrelated traumatic event (Shelby et al., 2008). Future studies should examine more closely the source of emotional numbing associated with pre-and post-surgical pain.

The results of the present study also support recent suggestions that avoidance and emotional numbing constitute separate PTSD symptom clusters (Asmundson et al., 2004; Foa et al., 1992). Concurrent emotional numbing, but not avoidance, was a significant predictor of pain disability at the 6 and 12 month follow-ups. In addition, avoidance symptoms, but not emotional numbing, pain disability or pain intensity, decreased significantly over time, from the pre-operative assessment to the 12 month follow-up. These findings are consistent with a recent study showing that pain severity and emotional numbing, but not the other PTSD symptom clusters, are significantly negatively related to role functioning in patients with ongoing pain subsequent to motor vehicle injury (Clapp et al., 2008). Taken together, these results support the suggestion that combining avoidance and emotional numbing into a single symptom cluster may not be the most accurate conceptualization of PTSS (Asmundson et al., 2004).

From a treatment perspective, there is very little published on co-morbid PTSD and chronic pain (Asmundson and Taylor, 2006). There is some evidence a cognitive behavioral perspective that addresses both disorders is essential; otherwise, due to mutual maintenance of symptoms or shared vulnerability factors, improvement when only one disorder is treated may be temporary or may yield only partial resolution of symptoms (Asmundson and Taylor, 2006). The de-coupling of pain and PTSD symptoms with time would argue for the early initiation of treatment. However, until we know more about the source and nature of the traumatic stressors the patients experienced it may be premature to make treatment recommendations.

There are several limitations to the present study notwithstanding its prospective and longitudinal design. First, we recruited a convenience sample and not a consecutive series of patients. This may have introduced a bias. Second, the sample size was relatively small. Third, we did not assess depressive symptoms which bear some similarity to the symptoms of emotional numbing. However, the association between depressive symptoms and the symptoms of emotional numbing is unlikely due to symptom overlap (Asmundson et al., 2004) in light of evidence showing that they are distinct constructs (Blanchard et al., 1998; Feeny et al., 2000; Kashdan et al., 2006). We did not assess the nature of the traumatic stressors that contributed to the PTSS at the three interviews and 
so we do not know the source(s) of the emotional numbing and whether these remained constant or changed over time. These limitations suggest a number of considerations for future studies that will build on the currently limited understanding of pain disability following lateral thoracotomy and the specific contributions made by pain intensity and emotional numbing.

\section{Acknowledgments}

The authors thank Dr. Maria Dzyuba and Aubrey Chiu for their help in recruiting and/or interviewing patients. J.K. is supported by a Canadian Institutes of Health Research (CIHR) Canada Research Chair in Health Psychology at York University. G.J.G.A. is supported by a CIHR Investigator Award. The study was funded in part from a CIHR New Emerging Team Grant \#PTS-63186 to G.J.G.A. and funds from the Canadian Foundation for Innovation, Ontario Innovation Trust and a CIHR Canada Research Chair in Health Psychology to J.K.

\section{References}

American Psychiatric Association. Diagnostic and statistical manual for mental disorders. 4th ed. Text revision. Washington, DC: American Psychiatric Association; 2000.

Asmundson GJ, Taylor S. PTSD and chronic pain: cognitive-behavioral perspectives and practical implications. In: Young G, Kane A, Nicholson K, editors. Causality: psychological knowledge and evidence in court. Springer: New York; 2006. p. 225-39.

Asmundson GJ, Frombach I, McQuaid J, Pedrelli P, Lenox R, Stein MB. Dimensionality of posttraumatic stress symptoms: a confirmatory factor analysis of DSM-IV symptom clusters and other symptom models. Behav Res Ther 2000;38:203-14.

Asmundson GJG, Coons MJ, Taylor S, Katz J. PTSD and the experience of pain: research and clinical implications of shared vulnerability and mutual maintenance models. Can J Psychiatr 2002;10:903-37.

Asmundson GJ, Stapleton JA, Taylor S. Are avoidance and numbing distinct PTSD symptom clusters? J Trauma Stress 2004:17:467-75.

Baker DG, West SA, Orth DN, Hill KK, Nicholson WE, Ekhator NN, et al. Cerebrospinal fluid and plasma beta-endorphin in combat veterans with post-traumatic stress disorder. Psychoneuroendocrinology 1997;22:517-29.

Bender JL, Hohenadel J, Wong J, Katz J, Ferris LE, Shobbrook C, et al. What patients with cancer want to know about pain: a qualitative study. J Pain Symptom Manage 2008;35:177-87.

Black EK, White CA. Fear of recurrence, sense of coherence and posttraumatic stress disorder in haematological cancer survivors. Psychooncology $2005 ; 14: 510-5$.

Blanchard EB, Jones-Alexander J, Buckley TC, Forneris CA. Psychometric properties of the PTSD Checklist (PCL). Behav Res Ther 1996;34:669-73.

Blanchard EB, Buckley TC, Hickling EJ, Taylor AE. Posttraumatic stress disorder and comorbid major depression; is the correlation an illusion? J Anxiety Disord 1998:12:21-37.

Cella D. Quality of life considerations in patients with advanced lung cancer. Semin Oncol 2004;31:16-20.

Chibnall JT, Tait RC. The pain disability index: factor structure and normative data. Arch Phys Med Rehabil 1994;75:1082-6.

Clapp JD, Beck JG, Palyo SA, Grant DM. An examination of the synergy of pain and PTSD on quality of life: additive or multiplicative effects? Pain 2008.

Crombie IK, Davies HT, Macrae WA. Cut and thrust: antecedent surgery and trauma among patients attending a chronic pain clinic. Pain 1998;76:167-71.

Dajczman E, Gordon A, Kreisman H, Wolkove N. Long-term postthoracotomy pain. Chest 1991;99:270-4.

Feeny NC, Zoellner LA, Fitzgibbons LA, Foa EB. Exploring the roles of emotional numbing, depression, and dissociation in PTSD. J Trauma Stress 2000;13:489-98.

Felmingham KL, Bryant RA, Kendall C, Gordon E. Event-related potential dysfunction in posttraumatic stress disorder: the role of numbing. Psychiatry Res 2002;109:171-9.

Fischer AA. Reliability of the pressure algometer as a measure of myofascial trigger point sensitivity. Pain 1987;28:411-4.

Foa EB, Zinbarg R, Rothbaum BO. Uncontrollability and unpredictability in posttraumatic stress disorder: an animal model. Psychol Bull 1992;112:218-38.

Glover H. A preliminary trial of nalmefene for the treatment of emotional numbing in combat veterans with post-traumatic stress disorder. Isr J Psychiatry Relat Sci $1993 ; 30: 255-63$

Gotoda Y, Kambara N, Sakai T, Kishi Y, Kodama $K$, Koyama T. The morbidity, time course and predictive factors for persistent post-thoracotomy pain. Eur J Pain 2001:5:89-96.
Hamner MB, Hitri A. Plasma beta-endorphin levels in post-traumatic stress disorder: a preliminary report on response to exercise-induced stress. Neuropsychiatry Clin Neurosci 1992;4:59-63.

Hanley MA, Jensen MP, Ehde DM, Hoffman AJ, Patterson DR, Robinson LR Psychosocial predictors of long-term adjustment to lower-limb amputation and phantom limb pain. Disabil Rehabil 2004;26:882-93.

Hanley MA, Jensen MP, Smith DG, Ehde DM, Edwards WT, Robinson LR Preamputation pain and acute pain predict chronic pain after lower extremity amputation. J Pain 2007;8:102-9.

Hayes C, Browne S, Lantry G, Burstal R. Neuropathic pain in the acute pain service: prospective study. Acute Pain 2002;4:45-8.

Hoffman L, Burges Watson P, Wilson G, Montgomery J. Low plasma beta-endorphin in posttraumatic stress disorder. Aust N Z J Psychiatry 1989;23:269-73.

Jensen MP, Ehde DM, Hoffman AJ, Patterson DR, Czerniecki JM, Robinson LR Cognitions, coping and social environment predict adjustment to phantom limb pain. Pain 2002;95:133-42.

Ju H, Feng Y, Yang BX, Wang J. Comparison of epidural analgesia and intercostal nerve cryoanalgesia for post-thoracotomy pain control. Eur J Pain 2008:12:378-84

Kangas M, Henry JL, Bryant RA. Predictors of posttraumatic stress disorder following cancer. Health Psychol 2005;24:579-85.

Kashdan TB, Elhai JD, Frueh BC. Anhedonia and emotional numbing in combat veterans with PTSD. Behav Res Ther 2006;44:457-67.

Katz J, Cohen L. Preventive analgesia is associated with reduced pain disability 3 weeks but not 6 months after major gynecologic surgery by laparotomy Anesthesiology 2004;101:169-74.

Katz J, Melzack R. Measurement of pain. Surg Clin North Am 1999:79:231-52.

Katz J, Jackson M, Kavanagh BP, Sandler AN. Acute pain after thoracic surgery predicts longterm post-thoracotomy pain. Clin J Pain 1996;12:50-5.

Kehlet $H$, Jensen TS, Woolf CJ. Persistent postsurgical pain: risk factors and prevention. Lancet 2006;367:1618-25.

Macrae WA. Chronic pain after sternotomy. Acta Anaesthesiol Scand 2001a;45:927-8.

Macrae WA. Chronic pain after surgery. Br J Anaesth 2001b;87:88-98.

Maguire MF, Ravenscroft A, Beggs D, Duffy JP. A questionnaire study investigating the prevalence of the neuropathic component of chronic pain after thoracic surgery. Eur J Cardiothorac Surg 2006;29:800-5.

Mountain CF. Revisions in the international system for staging lung cancer. Chest 1997;111:1710-7.

Mountain CF, Dresler CM. Regional lymph node classification for lung cancer staging. Chest 1997:111:1718-23.

Mylle J, Maes M. Partial posttraumatic stress disorder revisited. J Affect Disord 2004;78:37-48

Ochroch EA, Gottschalk A, Augostides J, Carson KA, Kent L, Malayaman N, Kaiser LR, Aukburg SJ. Long-term pain and activity during recovery from major thoracotomy using thoracic epidural analgesia. Anesthesiology 2002;97:1234-44.

Pearman T. Psychosocial factors in lung cancer: quality of life, economic impact, and survivorship implications. J Psychosoc Oncol 2008;26:69-80.

Perkins FM, Kehlet H. Chronic pain as an outcome of surgery. A review of predictive factors. Anesthesiology 2000;93:1123-33.

Perttunen K, Tasmuth T, Kalso E. Chronic pain after thoracic surgery: a follow-up study. Acta Anaesthesiol Scand 1999;43:563-7.

Pluijms WA, Steegers MA, Verhagen AF, Scheffer GJ, Wilder-Smith OH. Chronic postthoracotomy pain: a retrospective study. Acta Anaesthesiol Scand 2006;50:804-8

Pollard CA. Preliminary validity study of the pain disability index. Percept Mot Skills 1984;59:974

Senturk M, Ozcan PE, Talu GK, Kiyan E, Camci E, Ozyalcin S, Dilege S, Pembeci K. The effects of three different analgesia techniques on long-term postthoracotomy pain. Anesth Analg 2002;94:11-5.

Shalev AY, Peri T, Canetti L, Schreiber S. Predictors of PTSD in injured trauma survivors: a prospective study. Am J Psychiatry 1996;153:219-25.

Shelby RA, Golden-Kreutz DM, Andersen BL. PTSD diagnoses, subsyndromal symptoms, and comorbidities contribute to impairments for breast cancer survivors. J Trauma Stress 2008;21:165-72.

Tait RC, Pollard CA, Margolis RB, Duckro PN, Krause SJ. The pain disability index: psychometric and validity data. Arch Phys Med Rehabil 1987;68:438-41.

Tait RC, Chibnall JT, Krause S. The pain disability index: psychometric properties. Pain 1990;40:171-82

Taylor S, Fedoroff IC, Koch WJ, Thordarson DS, Fecteau G, Nicki RM. Posttraumatic stress disorder arising after road traffic collisions: patterns of response to cognitive-behavior therapy. J Consult Clin Psychol 2001;69:541-51.

Taylor S, Thordarson DS, Maxfield L, Fedoroff IC, Lovell K, Ogrodniczuk J. Comparative efficacy, speed, and adverse effects of three PTSD treatments: exposure therapy, EMDR, and relaxation training. J Consult Clin Psychol 2003;71:330-8

Weathers FW, Litz BT, Herman DS, Huska JA, Keane TM. The PTSD checklist: reliability, validity, \& diagnostic utility. In: Paper presented at the annual meeting of the international society for traumatic stress studies, San Antonio, TX; 1993. 